\title{
Can Reference Be Naturalized?-Notes toward an Integrational Causality
}

\author{
Daihyun Chung \\ Ewha Womans University
}

\begin{abstract}
As physicalisms of various kinds have faced difficulties in recent years, the time has come to explore possible alternatives, one of which is yinyang ontology. A yinyang theorist is expected to provide a plausible account of causation to replace the traditional notion of causation. The present paper is critical of the Humean tradition, which understands the relata of causal relations in terms of passive materiality so that humans use referential terms to describe causal relations constructively. But an alternative notion of reference is available according to which causal relata are active processors of the information with which they interact. On this latter view, humans use referential language to describe the structure in which the relata interrelate themselves so that the structure can be understood hermeneutically. Reference on this view is naturalized. In this article, I advance two arguments for this thesis, one concerning the informationality of states and the other related to the essentiality of properties.
\end{abstract}

Keywords: yinyang causality, hermeneutical reference, intentionality of states, causality as natural kinds, essential properties

How should contemporary philosophers respond to the success of modern science? There are at least the following three options: (1) to oppose any serious role for science in view of the negative consequences that science may bring to humanity; (2) to explain the metaphysics of the scientific worldview in terms of physicalism; and (3) to adopt a position that is neither for nor against science. The present paper explores the second of these options by considering whether physicalism may be replaced by yinyang ontology.

Physicalism and yinyang can be compared in terms of the notion of causality. While physicalism arose in response to dualism, it is still under its influence in taking causal relata as things which lack, not only mentality, but also power, activism, and agency. Yinyang does not posit things of the world in terms of non-communicable opposition, such as matter over mind, which requires different sorts of relations between causal relata. In what follows I examine the relations between causal relata that are allowed by yinyang. I propose that reference should be naturalized in order to move from the Humean concept of causation to yinyang causation. In support of this thesis, I argue that states are informational and that causal properties are essentially natural kinds.

Daihyun Chung, Ph.D., Professor Emeritus, Department of Philosophy, Ewha Womans University, South Korea; main research field: Philosophy of Language, Asian Philosophy, and Analytic Metaphysics.

Acknowledgement: This work was supported, in part, by the National Research Foundation of Korea Grant funded by the Korean Government (NRF-2010-342-1-A00005). Drafts of the present article, Korean or English, were read at the Institute of Philosophical Studies, Korea University, May 30, 2014, and at the 2nd CCPEA (Conference on Contemporary Philosophy in East Asia), August 28-29, 2014 (Kyoto University). I thank the participants of those meetings for their helpful comments and constructive criticisms. I am especially grateful to Professors John McGuire, Sangkyu Shin, and Sa-soon Youn for their encouragement, stimulus, and guidance to improve my paper. 


\section{Introduction: From Linear Causality to Yinyang Causality}

\subsection{Passivity in Linear Causality}

Hume (1951; 1966) characterized causality in terms of notions like contiguity, constant conjunction, temporal priority, and necessity so that his notion of causality may be called a "linear causality.” Mackie (1965), who thought that causality is extensional and subject to the language of the first-order logic, offered a physicalistic analysis of the concept. For example, the fire which broke out at Daeyongak Hotel in Seoul on December 25, 1971 and resulted in 163 deaths was caused by combustion sparks in a liquefied petroleum gas container. According to the analysis, the sparks were neither necessary nor sufficient for the fire, but were rather a necessary part of one sufficient condition for the occurrence of the fire.

The notion of linear causality faces certain conceptual problems, one of which is the assumption that the objects or events related through linear causality are materially passive. The complex event that was a sufficient condition for the Daeyongak fire consisted of, among other things, combustion sparks, oxygen, inflammable materials, and dry air, none of which were actively inter-related. The physicalistic conception of causality based on the language of first-order logic dictates that nothing material can be active; material objects on this view are passively mechanistic, being nothing more than spatio-temporal points. What is mysterious is how such diverse elements constitute the complex event that is able to produce the fire causally. If those elements are nothing more than mechanistic parts, where does the mechanistic power come from? How could the complex event become an agent for the fire? Physicalists tend not to ask these questions; instead they assume that only humans are agents and that all other material things are passive constituents of the world.

Let us pause to consider a distinction, which is central to my argument, between physicalism and naturalism. On the one hand, physicalism is understood as an ontological-methodological thesis that all things or phenomena are physical or can be explained in physical terms. One consequence of this thesis is the idea of super-venience, the idea that two objects cannot be different without being physically different. What is important is how to characterize the term "physical." Traditionally, a physical object has been regarded as a substratum that has properties like extension, shape, and movement as its constitutive elements and also has the properties of being inert and senseless. Quantum mechanics tends to decrease the importance of the former and yet maintains the significance of the latter. What it means for an object to be physical on this view remains to be explained. Theories of events, such as Jaegwon Kim's exemplification of a physical property or Donald Davidson's physical description of an event are based on Cartesian dualism. On the other hand, naturalism may be taken as an ontological-methodological thesis that phenomena can exist and be explained without any non-natural intervention. Physicalism and naturalism share the same interest in stressing the importance of scientific methodology and excluding super-naturalism, but they are different in implementing their methodologies. For example, physicalism leans toward the causal exclusion hypothesis that all events have physical causes, while naturalism does not commit to that hypothesis and remains free from notions of inert or senseless matter.

\subsection{Causality in Yinyang Ontology}

As with many traditional ideas, the concept of yinyang is somewhat vague and confused. The concept has been used in at least the following four ways. Firstly, the statement that men are yang and women are yin expresses a substantial view of yinyang. Secondly, the view that objects come to have different properties of yinyang according to their functions in context may be classified as a functional theory of yinyang. Thirdly, the 
interpretation that yin and yang are not real properties of objects but rather their adverbial aspects emphasizes the modal character of yinyang. Fourthly, and most importantly for the purposes of this article, the idea that all things in the world including their parts and properties maintain essential/contingent relations to each other may be called an "organic conception of yinyang" (Chung 2001). If we accept this conception, then yinyang refers to the essential properties of objects in the system we live in, where if one of them behaves in a concave (凹) fashion then the other will behave in a convex (凸) fashion. Yinyang ontology does not limit itself to the relations of two particular objects. Rather, it relates one object to all other things in the universe. All things in the system enter into the relation of mutual sympathy and the system may be represented in terms of the ways in which these things behave. Furthermore yinyang connects various temporal stages and dimensions, and the process may consist of transitive processes and repetitive loophole movements (Chung 2001, 12-22). ${ }^{1}$

Understanding yinyang in organic terms helps to explain ordinary experiences. The linear theory of causation presents as typical cases of causation examples such as John's hitting the baseball caused the breaking of a glass window in Mary's room, understanding the relation between the two events (the hitting of the ball and the breaking of the glass) in terms of linear temporality. But the yinyang notion of causality would rather take as exemplary an ice-cube cooling the water in a glass and the water melting the ice-cube; it would regard the relation between these two events (the ice-cube's cooling the water and the water's melting the ice-cube) as being simultaneous and mutual. Recent metaphysical arguments support this perspective.

For example, Martin (2008) assumes that causality is simultaneous and ubiquitous. In the case of two cards leaning against each other, he claims that one card's standing up is not a cause of the other card's standing up; rather the two cards' standing up is both mutual and simultaneous, one causing the other and vice versa. Furthermore, Martin claims that this sort of mutual causality is ubiquitous. Of course, there are more dispositional properties than these two which are engaged here. For example, properties like the table's supporting the cards, or a window's being open, or a wind's blowing but too slightly are involved in the events of two card's leaning against each other. However, they exhibit no manifestations that they are there, as they are constrained by those dispositional properties. On the surface, only two events appear to be involved in examples such as the baseball's breaking the window or the combustion sparks' causing the hotel fire. But any causal event is involved with all other properties of all objects in the world, most of which generally keep a low profile, though they are prepared to respond in appropriate circumstances. It is in this sense right to say that this is "a busy world."

Another argument for the thesis of mutual causality has been advanced by Molnar (2003) who claims that a type of causation constitutes a natural kind. For example, events are typically not what a power manifests but rather what a number of different powers in combination manifest. In a similar vein, many genes will typically contribute to the production of a trait, which is called "polygenic.” The converse of polygeny is "pleiotropy," which refers to the condition in which one gene contributes to the production of many traits. Properties and powers found in genes are polygenic and pleiotropic at the same time. Such dispositions of a gene are characteristic of a natural kind. Of course, the dispositionality of a gene is isomorphic with respect to the manifestation of the gene. For the identity of a gene's dispositions is obtained by observing the structure behind the gene's manifestations. The properties in a gene's dispositions or manifestations behave either in a concave or convex fashion, fitting oneself to the other, maintaining the order of agreement between the structure of a gene's disposition before a stimulus and the gene's manifestation after the stimulus. The observation that causality is a natural kind is a basis for explaining yinyang causality. 


\subsection{Dispositions as Intentionality}

Since the linear view of causation assumes that causal relata are passive in nature, yinyang theory should be able to provide an account of their active agency. As is stated in The Doctrine of the Mean (Confucius 1971), things in the world have agency. The doctrine centers on the conception of cheng (誠), which may be summarized in terms of the following five propositions: (1) the integrationality of an entity is its power to realize its embedded objective in contexts in which it interacts with all others; (2) the word "mind" refers to the ability of all entities of a complex degree in processing information; (3) integrationality is a power of minds, not only of humans, but also of all things; (4) if evolution reflects a history of species struggling to survive, then history represents an evolution of life forms that are intelligent and just; (5) integrationality is the realization of what can be the best in a given situation in which a subject is involved with its surroundings (Chung 2008a; 2008b). The integrationality or cheng power of things has intentionality, which facilitates that power's execution. ${ }^{2}$ Thus, all things in the world have a metaphysical basis for their powers of integration. The notion of "fitting" in the Korean language illustrates this concept of integration (Chung 2008b).

Fortunately, the integrational metaphysics found in East Asia is reflected in some recent works in the Western analytic tradition. Molnar (2003) takes the dispositions in things as intentional, going beyond the dualistic confines of mind and matter. He sees continuity or parallels between mental and physical intentionality with respect to directionality, inexistence, ambiguity, referential opacity, and non-truth functionality. Molnar notes that just as lactation is a biological function of a higher dimension, so too intentionality is a brain function of a higher type, and he discusses how dispositions form the basis for causal intentionality. Shani (2012) also notes the characteristics that are shared by mental and non-mental intentionality such as projectibility, internal connection, modality, aspect relativity, and proper essentiality. He asks whether the sharing of those characteristics is accidental or rather whether physical intentionality is the ontological base from which mental and social intentionality might have evolved. He argues that non-mental intentionality is continuous with, and necessary for, mental intentionality.

The concept of a disposition is in need of clarification at this point. Philosophers such as Hume and Carnap recognize the concept of a disposition but their views are based on a distinction between observable and unobservable properties. For example, solubility is a disposition when it is not observable, and the dispositional property of "solubility" must be reduced to an observational property of "being solved when put into water." But recent metaphysical accounts of dispositions are interested in dispositions in light of whether the properties are involved with human perception, not whether the properties are observable. As John Heil (2003) claims, properties or qualities are all objects of perceptions and properties cannot be objects of perceptions without power. Further, the individuation of properties cannot be separated from the individuation of manifestations of properties. Therefore, powers of properties become dispositions of properties and all properties are dispositions.

\subsection{Need for a Naturalized Reference}

Why does one need a notion of reference in the context of causation? Without reference, an explanation to a why-question is impossible. For any explanation to a why-question is constructed in terms of reference to other elements including general statements, which all involve reference to individuals.

But what is reference? Traditionally, reference has been understood as the name of a particular relation between an expression and an object such that the expression stands for, represents, denotes, designates, or refers to the object. Reference is a tool by which people can talk about an object. This traditional notion of 
reference is of interest because it requires a special relation between humans and objects, one in which humans are active agents and objects are passive recipients. Objects are not given any role to play in the ways in which humans refer to objects. Humans grant names to objects so that they may exist meaningfully for them and in doing so they construct the referential relation between an expression and an object. The traditional notion of reference seems to bring with it a dualistic metaphysics. This notion may be called "reference 1."

What then is "reference naturalized?" One may expect that the notion of reference based on integrational yinyang should be different from reference 1. If the world is integrationally organic rather than dualistic, then reference, as an act by which humans give names to objects in the world, should be understood differently. Parts of the organic world should be recognized as agents that execute their powers of communicating with each other in various ways. Humans then come to recognize objects as active processors of information and interpret this active processing, which is how humans refer to objects. On this view, then, implicit in the notion of reference is the idea that the objects themselves play an active role in communicating with each other. We may call this notion "reference 2."

Since reference 1 is based on dualism and reference 2 arises out of yinyang ontology, they have different conceptions of causality. Physicalism, which is consistent with reference 1, holds that relevant causal relata stand in a nomological relation and does not seek to understand how they are inter-related. Reference 1 has been used to denote objects insofar as they are regarded as causal relata. Reference 2 on the other hand takes causal relations as involving more than two events. Yinyang causality should be holistically constructed since yinyang ontology is organic. Thus, any notion of reference must relate to what is there organically, which is why reference 2 is just an interpretation of what is organically given (Molnar 2003).

Naturalized reference is consistent with naturalized intentionality but is to be distinguished from naturalized epistemology. ${ }^{3}$ The former presupposes yinyang ontology but the latter is founded on the physicalism of science. Physicalism understands the world as passive and lacking intentionality, but yinyang assumes an organic world, all parts of which are intentional. Both of these perspectives use terms like "naturalism" and "naturalized," but they are positioned in opposing ontologies. When yinyang talks about the organic world, it understands it at the level of causation as the totality of conditions which are positive and negative (Mumford and Anjum 2011, 11-13). This makes possible the hypothesis that every previous event in the universe counts as a cause of every later effect.

Since yinyang causation is a plausible and in some sense preferable notion to linear causation, it is important to explain how yinyang reference can be formulated. Yinyang takes for granted that dispostions, physical intentionalities, power, and chi (氣) are given, holding that all things in the world are of integrationality (誠). Since causal relations are natural kinds, references are believed to be natural in the context of the causal phenomena. For this reason, the notion of naturalized reference needs to be analyzed. In what follows, I will provide two arguments for the thesis of naturalized reference, one from the informationality of states of affairs and the other from property essentialism.

\section{Informationality in States of Affairs}

\subsection{Informationality in Natural Kinds Causality}

When physicalism constructed its notion of causality, it closely followed the dualistic view of matter as lacking intentionality and thus as being passive. This resulted in the constructive notion of reference, reference 
1. But the yinyang notion of reference 2 is the interpretation of the workings of the integrational structure among the elements natural kinds of individuals. Let us look further into this idea that causal relations are natural kinds.

Natural kinds are ordinarily found in biological entities, such as cows or potatoes, and in materials such as gold and water. In order to find out how these natural kinds behave, we need to observe them scientifically. Scientific observation necessarily involves interpretation. For example, chemists make a molecular theory to investigate material elements, and they interpret the classification of molecular elements and atomic numbers and so on out of their theoretic investigations. When they say "water is $\mathrm{H}_{2} \mathrm{O}$," "caffeine is $\mathrm{C}_{8} \mathrm{H}_{10} \mathrm{~N}_{4} \mathrm{O}_{2}$," or "gold is Au," they are offering an interpretative reference.

If causal relations are a type of natural kinds, they should behave more or less like ordinary natural kinds. If it is possible to show how to make an interpretative reference to causal relations this will be an argument in support of the thesis of naturalized reference. In order to demonstrate that causal relations are structured and systematic and that they function as natural kinds, one needs to show that causal relations have informational structure. It is to this topic that we now turn.

\subsection{Information Localization}

On the surface, there seems to be a tension between yinyang's organic integrationality thesis and the causal thesis that seems to limit causal relations to binary causal relata. The tension is serious only if one understands the causal relations in the traditional sense. But yinyang causality does not limit the relation only to the pair of objects or events directly involved. The notion of information localization is useful for understanding the relation between the two and removing the apparent tension. In the first place, consider the localization of brain functions. The human brain has localized functions such as sight, hearing, sensation, movement, language understanding, and aphasia. Yet, these localized areas exchange information with each other through the nervous system, which activates, connects, and integrates them into a unified perception (Kim 2011). ${ }^{4}$

The notion of brain localization is relevant to the informationality of states of affairs. Three points should be noted in this context: (1) the pair of causal relata may be taken as "information localized," where two events are inter-processing the relevant information; (2) but other states are also information localized even when they are working in silence; (3) as the nervous system integrates all the localized information into a unified whole, states of affairs in the system are integrated into an organic whole. States of affairs are themselves dispositions and these can be accessed through a dispositional analysis (Mumford and Anjum 2011; Marmodoro 2010). Causes do not necessitate their effects; rather they are dispositions toward those effects, since their effects can be constrained by other additional dispositional forces. Those forces are working additionally or subtractionally just as vectors work with directions and sizes. This model allows for a situation in which each of causal modality, the manifestational properties of powers, the subdued causality of powers, and causal probabiliity is activated. On this view, the structure of information is localized and exhibits manifestations, either occurent or dispositional.

\subsection{Integrationality of States}

The proposition that states are informational is plausible but in need of conceptual clarification through a study of its ontological structure. Such conceptual clarification may be approached by examining the properties 
that constitute the elements of those states. Traditionally, states have been understood as bundles of passive properties. If we can see how properties are powers, integrational or fitting with others, then we will be in a better position to appreciate the main thesis of our investigation.

The idea that qualities are separate from power has a long history. Galilleo demanded that science should focus on the primary properties such as size, shape, and quantity, but not on the secondary properties such as colour, sound, taste, and smell, which he thought are not physical. However, Descartes reversed Galilleo's order of primacy of the physical to the mental, advocating a dualism of the thinking mind and extended matter. Physicalism followed the metaphysics of Cartesian dualism in allowing physical elements to have causal properties but did not attempt to explain how these causal properties work to produce results. Recent developments in dispositional metaphysics have significance for yinynag ontology. For example, consider magnets attracting iron filings. If physicalism bestows the magnet with the active agency to attract while treating the iron filings as passive recipients, the descriptions of the roles of the agent and the recipients should be familiar to the followers of Descartes. But dispositionalism is ready to grant active agency both to the magnet as well as to the iron filings, and the relation of reciprocity between them would not hold unless they both possessed the property of permeability. Properties and powers on this view are one and the same (Heil 2010).

Why are properties so mutually responsive to each other; in other words, what is it that makes them integrational? Dispositonal metaphysics provides some guidance on this question (Martin 2008). First, the properties of an object have internal connections with the dispositions of the object. Simple properties like "being four-sided" are involved with more complex properties like "being square," distinct properties like "being frozen" are related contingently and nomologically with properties like "being expanded," and some properties are necessarily related as in "being equilateral" and "being equiangular." Second, properties like "being soluble" and "being solvent" are properties of different objects like salt and water; they exhibit mutuality through their shared manifestation in appropriate circumstances. Third, "two cards' standing up and leaning against each other" shows that the manifestation of their mutuality and simultaneity is a case of ubiquitous phenomena in nature.

Finally, let us consider what it means for states of affairs to be informational. For this, states of affairs should not remain atomistically separated but should be organically connected so that their fittings to each other can be integrational. Williams (2010) discusses this notion of fitting. The manifestations (Ms) which are observed in nature are the result of the mutual interrelated workings of those properties of individual objects or of their parts on certain stimuli. And what are called "stimuli required for manifestations" are there mostly in the form of a presence of powers of properties, but could also be there in the form of absence of them. Of course, "hitting a baseball” and a "window's being broken” are a mutually fitting pair, but there are other forces of properties in the surrounding objects or their elements which remain silent. We need to be reminded of these apparently non-obvious forces as well. Then, the fitting condition of an event contains not only the pair which can be observed but also the surrounding forces which remain silent.

\subsection{Holism of States}

In order to demonstrate that states are informational, it would suffice to show that states are organic or holistic. If information is linguistic, then insofar as linguistic meaning is holistic, information is holistic. And if states are informational, then they are holistic, being transitive. States' being holistic means that properties of 
objects or of their parts are powerful and, furthermore, that they are structured in a way that they are fitting partners. For an example, the "turning of a key" and the "turning of the lock" are properties of my act of opening of my apartment door. The holism of these kinds of properties is ubiquitous throughout nature.

Williams (2010) analyzes the holistic character of states more concretely. He offers a thought experiment as to how powers of properties work fittingly in the context of causality. Suppose that all the information of the properties of states is packed into the power and that powers are classified according to a blueprint. Then the manifestations of all the causal results can be accessed by examining all the possible sets of mutual pairs. This is analogous to building with puzzle pieces. It is no surprise that when puzzle pieces are arranged according to a blueprint, all the pieces turn out to fit the board. The result of packing all the information of states into the power means that each property found in states carries in it the blueprint by which it fits with all the other properties in the universe. The properties of a fitting pair work together but it is important to recognize that the fitting pair does not tell the whole story.

The holism of states can be seen through the essentialities of properties. Properties of fitting pairs are, not accidental to each other, but rather essential for the fitting relation. For example, since it is necessary that two atoms of hydrogen combine with one atom of oxygen to form water, hydrogen's having 2 atoms and oxygen's having 1 atom are essential properties of water. It is necessary that water is $\mathrm{H}_{2} \mathrm{O}$, but the necessity of the proposition is not limited only to the modality of the relations that the two referents of the sentence denote. The proposition rather implies a wider modality which is prescribed in the blueprint in which the two properties are related to each other essentially. However, the two properties are also related to all the other properties in the world, although not manifestationally. Manifestations of powers of properties may appear separately on the surface, but they are interrelated holistically at a deep level. The holism of states is constituted by the integrational powers of all the properties there are.

\section{Essentiality of Properties}

\subsection{Essential Properties and Causality}

What are essential properties? A Kripkean example may help to explain what it means for an object to have an essential property. Barack Hussein Obama II is the 44th President of the USA. This is true and accidently true. The property described by "being the 44th President of the USA" is an accidental property which is realized in Barack Hussein Obama II. It is possible that John McCain might have been the 44th President of the USA, or that Obama II might not have been in the politics. But Barack Hussein Obama II, born August 4, 1961, is the only son of Mr. Barack Hussein Obama Sr. and Ms. Stanley Ann Dunham. If this is true, then it is necessarily true. The property referred to by the expression "the only son of Mr. Barack Hussein Obama Sr. and Ms. Stanley Ann Dunham” is an essential property realized in Obama II. The fertilized egg formed by the sperm of Mr. Barack Hussein Obama Sr. and the ovum of Ms Stanley Ann Dunham could not be other than Obama II. For there is a unique causal chain which started at the joining of the sperm and the egg and went through the conceptus, the embryo, the fetus to grow to be the adult. Supposing that this is the way in which we understand what an essential property is, then naturalized reference is what is obtained through an interpretation of what an essential property is, not by a human intervention into a natural language.

Let's consider the sentence, "The ice cube cools water in the glass and the water melts the ice cube." "The ice cube's cooling the water" and "the water's melting the ice cube" are properties, constituting mutual and 
simultaneous causal interrelations, manifesting their causal powers. These two properties maintain separate dispositions but work together as a fitting pair to bring out the causal manifestation. Two properties work on each other and become essential properties for the manifestation. They are inter-related to all the properties in the world, but each of them only becomes one of a fitting pair under appropriate conditions. Two properties thus refer to each other when they communicate with each other in their essentially causal inter-relation. Humans then come to interpret their essentially causal referring relations.

How is it possible that two properties of a fitting pair refer to each other? Mumford and Anjum (2011) offer guidance on this issue. For example, "the ice cube's cooling the water" and "the water's melting the ice cube" are said to have the function of selecting each other but no others to which they are exposed. These two properties are directed toward each other, have tendencies to search for each other, and favor each other. The causal powers and dispositional connectivities for their manifestations cannot be reduced to other terms.

\subsection{Further Considerations on Properties}

Essential properties were illustrated in the example of the origin of Barack Hussein Obama II. They can be also shown by other means. Let us try a thought experiment. Going back to the case of the water glass, suppose that the ice cube does not cool the water in the glass. Then we can infer that the situation in the water glass is not normal. Abnormal situations are those in which, for example, some elements in the glass block the ordinary workings of the ice cube's solubility or the water's catalysis. But if one cannot find empirical evidence that supports these abnormal cases, then one may infer that the initial supposition is wrong-that the ice cube does not cool the water in the glass. Thus, the ice cube in a normal situation cools water in a glass necessarily. The ice cube's cooling the water is the essential property of the ice cube.

Essential properties also receive support from the very notion of reference. Consider, for instance, the following two sentences:

(1) Bachelors are unmarried men;

(2) Ice cubes cool water.

Ordinary intuitions suggest that (1) is based on the meaning of the word "bachelor" and (2) is about a property of ice cubes. The former is purely semantic, and based on norms of the linguistic community, but the latter is about a natural kind, which says that an object exists. Put differently, the former is an analytic sentence and the latter is a synthetic sentence.

But Quine rejects the distinction between analytic and synthetic sentences, supposing that necessity or essence is a function of how an object is described. As for the same natural kind of human beings, one may describe it either as "human" or "featherless biped." But the sentence "humans are rational" is analytic and necessary while "featherless bipeds are rational" is not. Similarly, while "featherless biped are two-legged" is analytic and necessary, "humans are two legged" is not. Quine thinks that one cannot distinguish between speaking about objects and speaking about languages, for existence according to Quine is nothing but the value of a bound variable. If systems of first order logic differ, the values of variables will differ, resulting in a plurality of systems. Thus, Quine proposed a radical version of the notion that comparison between theories is incommensurable, one which denies any notion of essential properties.

I would take Quine's account of reference as a theory of constructive reference. It is true that there are many cases of constructive reference among human references but it is not true that all of them are constructive. Primitive references are not obviously constructive. Consider again Quine’s argument. Quine speaks of “the 
same natural kind of human beings." He does not pay a close examination to this phrase, but it plays an important role in his argument. Why did Quine ignore the obvious fact that this "same natural kind of human beings" has different references? If he could have seen this fact, he surely would have also recognized that many human references are interpretive references about that same thing.

The essential properties of an object are modalities which are primitively given and which cannot be analyzed away, neither the essential properties of Barack Hussein Obama II, nor of water. This is one reason why it is difficult to define a causal notion (Mumford and Anjum 2011). On the one hand, such definitions fail if the notion of modality is not included. On the other hand, including the notion of modality makes the definition circular. Thus, essential properties necessarily refer to each other in a fitting pair in order to maintain their modal status.

\subsection{Essence and Counterfactuals}

The last argument for naturalized reference is what may be called the argument from counterfactuals. If the dispositional universe is holistically modal, then the reference by which we interpret those modalities is nothing but naturalized reference. What is a counterfactual sentence? While some people assume a descriptive metaphysics which claims that factual worlds are only contingent, an alternative is to take a counterfactual sentence as a metaphysical tool to enable a thought experiment such that if things were different, facts might have been different. If the world has such a modality, then a counterfactual sentence can be employed to show that an interpretive reference takes place in the connective structure of modally dispositional powers.

Let us return to the example involving the fertilized egg that became Barack Hussein Obama II. A counterfactual sentence is here useful to show one of Obama's essences. Let us call the fertilized egg by the name of "ObaDun" and regard the fertilized egg as a person, ignoring for the moment the moral status of embryos. Clearly, Barack Hussein Obama II, and ObaDun are identical. For they both came from union of the very same sperm and egg. Some counterfactual sentences arise in this context: "Might Obama II have been born from another man's sperm or another woman’s egg?”; "If the sperm were united with an egg other than that egg, might the result not still be ObaDun or Obama II?”; “If the egg were united with a sperm other than that very sperm, might not the resultant fertilized egg still be ObaDun or Obama II?” These counterfactual sentences can give rise to various debates. What is important is to see that even without the name "ObaDun" one can make an interpretive reference when talking about counterfactual situations. Humans can participate in the world naturalized by means of naturalized reference.

\subsection{Implications of Naturalized Reference}

The present article has sought to describe a concept of reference on which the theory of yinyang ontology may be based. This attempt is preliminary and has at least two limitations. The first is that the idea of naturalized reference is itself not natural, for how can one naturalize the linguistic notion of reference, which is by definition non-natural? Thus, it is necessary to show that dispositions of properties can be active agents of information processing. But it may seem difficult to accept the idea that properties of things are intentional. Of course, there is a difference between mental and physical intentionality, for human intentionality is the supreme form of intentionality in the history of evolution. But what is important is to see that there is continuity between human intentionality and physical intentionality. It is therefore plausible to suppose that human intentionality is a part of the evolutionary process. 
The second limitation of the present study is that it has until now remained silent on the question of how to connect the concept naturalized reference with the traditional concept of reference. If the traditional concept is constructive while naturalized reference is interpretive, exactly how are the two concepts related? However, this question can be answered. The continuity and separation of the two notions of references can be discerned by examining their metaphysical dimensions and logical character.

Logically, the two notions of reference will have only qualitative differences. For the logical status of reference, whether it is reference 1 or reference 2 depends on the frame chosen by the speaker. When Frege spoke of "sense" as an object of a third world and Kripke talked of objects that exist in all possible worlds they shared interpretative elements in their notions of reference. On the one hand, the reason why the properties of an object cannot be contingent is that the sense by which an expression refers to the object is an element of the objective third world. Even though Kripke's notion of rigid designator appears to be a human construct, the expression "rigid designator" indicates an object that is referred to with the same name in all the possible worlds where it exists. On the other hand, naturalized reference is the hypothesis that reference must reflect the properties of an object found in the natural world. Thus, at the logical dimension, there are elements of interpretation in varying degrees in human acts of reference, whether they mention the third world or possible worlds or the natural world. This indicates that there is continuity between the traditional concept of reference and the concept of naturalized reference.

Metaphysically, the notions of reference in Frege and Kripke are notions of reference by which speakers substitute objects, taking references as something standing for the objects so that they can talk about them. Frege's object is a collection of properties that are known to speakers and Kripke's object is rigidly designated by speakers. Naturalized references are called "references" but these are themselves objects and speakers approach them as both investigators and interpreters, rather than as regulators or creators. This is where one can see the discontinuity between the two concepts of reference.

In spite of such limitations, the present discussion has some significance. In the first place, consider some of the recent achievements of science and technology. The development of computing helps to understand both the continuity and discontinuity between analogue and digital informations. These two are discontinuous in that one is a mode of objects by which we perceive while the other is a mode by which essences of an object are constituted. But these two notions are also continuous in that they are translatable to each other at certain dimensions of their appearances. One may say that references are already naturalized in this trend of digitalization. The processing of information that takes place in astrophysics and microbiology overcomes the traditional discontinuity between the analogue and the digital in terms of a mathematical continuity between them.

Physicists like N. Bohr and W. Heisenberg helped to found a theory by which the spectrums of lights are interpreted so that various properties of things or astronomical bodies can be calculated. Then the Hubble telescope enabled one to digitalize the analogue lights into what is called HUDF (Hubble Ultra-Deep Field), which shows that one galaxy contains thousands of trillions of stars and that there are over a thousand trillion galaxies. Biologists recently have also made revolutionary advances. F. Sanger designed what is called "Sanger sequencing," which digitalized DNA analogue ATCG, and allowed scientists to read the information of life digitally, enabling the characterization of a life as a "a digital file.” This advance in turn led scientists like J. C. Venter to propose synthetic genomics as a means of synthesizing living organisms in order to get, for example, biofuels (Church and Regis 2012). 
A second important dimension of the present article relates to the history of philosophy. In the last several centuries, dualism and physicalism have been influential in philosophy. But it is now time to reevaluate what it means to hold one of these perspectives. Are there any alternative perspectives? East Asian philosophy has been largely unexplored since its languages have not been consistent with each of the two grammars resulted from the tradition of Cartesian dualism, namely, analytical physicalism and existential phenomenology. Fortunately, however, yinyang ontology has found some recent support in the metaphysics of dispositions and can now make some contribution to contemporary philosophy.

\section{Yinyang Metaphysics and Disposition Metaphysics}

It is natural for one to come to have various questions as to what was proposed in the three chapters above. For what was claimed there looks to be too sketchy to show the plausibility of an ambitious thesis that a yinyang notion of causation is an alternative to the dualistic notion of causation. I would limit this chapter to discuss just four questions among many, questions about the epistemological and ontological relation between yinyang metaphysics and disposition metaphysics (4.1), about the yinyang metaphysics and semantics of naturalized reference (4.2), about a reason to introduce the notion of essential properties into talks of semantics (4.3), and about non-dualism and non-symmetry in yinyang causation (4.4).

\subsection{Epistemologies and Ontologies of Two Metaphysics}

I defended the yinyang notion of causation by appealing to dispositional views of Martin and Molnar that the relata of causation have agency and potentiality to exhibit mutual interaction. Then, it is reasonable to ask for a reason to stack the yinyang notion upon the dispositional one. What is a benefit to call for the yinyang notion if the dispositional metaphysics can do all the works which are required to maintain the yinyang thesis? Certainly it does not appear to be economical to juxtapose two metaphysics, if they are equivalent.

I don't think that two metaphysics are identical. There are areas where they are complementary to each other but there are cases where their interests do not coincide. I would point to two cases in which they may diverge. The first case is that their primary epistemological questions are different from each other and the second is that scopes of their ontological units are different. Let's start with the first case. These two metaphysics share one question, namely, what are natural phenomena, not asking questions about supernatural. But their primary questions are different, where the yinyang metaphysics ask what is the integrational principle for all the things as there are and the disposition metaphysics ask what is the logic of dispositions in properties of objects.

I am inclined to say that the yinyang metaphysics is a world view which derives from the top whereas the dispositional metaphysics is a philosophical theory which starts from the bottom, our concrete questions about dispositions. The relation between two metaphysics may be said to be analogous to the relation between naturalism and physicalism. Many can agree that naturalism is a metaphysical view about the world from the top whereas physicalism is a philosophical theory which tries to explain a concrete relation between mind and body. That is, naturalism asks for the natural closure that physical events are determined by natural laws which are embedded in natural conditions whereas physicalism claims the physical closure that physical events are determined by physical laws attached in physical conditions (Won, C. 2013; 2014).

Two metaphysics are basically an internalist metaphysic, taking the same natural ontologies and no transcendent ones. But they take the ontological units of their interests differently. Disposition metaphysics take properties as their targets of study, assuming that properties are exemplified in objects but they are the ones 
through which objects are known and by which causal relations are linked and activated. Yinyang metaphysics is a comprehensive ontology where the continuity thesis holds among all the units of being. It covers levels of beings which are physical, chemical, biological, ecological, personal and it includes dimensions of being which are atomic, individual, aggregate, social, national, global. Those units of being are independent, maintaining their own integrity. And those units of being are continuous with each other, and yet each of them is not reducible to unit of other level or dimension. One can say that inquiries in disposition metaphysics are bottom up where yinynag metaphysics is top down.

\subsection{Yinyang Metaphysics and Semantics of Naturalized Reference}

Some people may read from what I wrote above that I offered a metaphysical claim that every object in the world informationally interacts with each other and a semantical claim that reference obtains by interpreting this informational structure in the world. Then, it is reasonable to question the semantical claim, by saying that a speaker needs not to interpret such structure in order to refer to things in the world. For, the causal theory of reference clearly illustrated that all a speaker needs to refer by a name successfully is to be merely connected to a chain of socio-historical structure in which the name in question is used.

Let me clarify what I wrote above. I meant the yinyang metaphysical thesis to picture that every object has its own agency and that every object in the world informationally interacts with each other, and the semantical thesis that we humans interpret this informational structure in the world where each object refers to each other as they informationally interact. Then, reference does not obtain nor it is created by a speaker's interpreting the informational structure. References are already there whenever events or properties call each other and interact to each other. This is the momentum where I tried to demonstrate that the notion of reference is to be naturalized without human intervention. Therefore, a speaker does not need to know anything about the informational structure in order to refer successfully. The notion of interpretation was introduced to show that references are there already and that some references do not require human intervention.

The notion of reference 1 also needs to be clarified. For, the notion seems confusing since it includes not only the description theory of names but also the causal theory of names. Of course, those two theories of names are different. For, names for one are indirectly referential through mediation of sense of names, and names for the other are directly referential through causal chain of uses of names. In spite of their claimed differences, I see what they share. It is that reference is given by humans in the way of intervention. Both do not recognize that some references are there naturally. Since they do not think that events or properties are not informationally active agents, they have to create references so that they can talk about them. This provides a basis for the notion of reference 1. It allows the contrast with reference 2 which recognizes that some references are there naturally as events interacts informationally with each other before human intervention.

\subsection{Essential Properties and Semantics of Reference}

In the context of my exposition of what are essential properties, I said "naturalized reference is what is obtained through an interpretation of what an essential property is, not by a human intervention into a natural language.” This sentence might be taken to claim that for a subject to refer to some object, she must know the essential properties of the object or at least have some belief on them. If this is true, then the claim is definitely too demanding. For, we usually refer to something, for example, a cat, without knowing the essence of the cat nor having some beliefs on its essence. 
It is important to notice that "obtained" in the passage is ambiguous to allow two readings. The initial reading above tends to imply that naturalized reference is to be given or created through an interpretation of what an essential property is. But this is not what I intended to say. What I meant the passage to convey was that naturalized reference is to be accessed or reached through an interpretation of what an essential property is. The sentence in question is given at the conclusion of an analysis for what an essential property is. The relation between naturalized reference and essential property is such that reference is there already naturally in the causal interactions among events without human intervention and naturalized reference is accessible through our investigations of essential properties involved in the causal interactions.

What role does the notion of essential property play here in the discussion of naturalized reference? The discussion of ambiguity above seems to help to see the picture more clearly. For, a distinction needs to be made between an essential property of an object described by a general term and an essential property of a relation denoted by a causal term. In the case of the former, for example, water is known as having the property of being $\mathrm{H}_{2} \mathrm{O}$ as its essential property and cat is not yet known to have a particular essential property if any. Water or cat is already known to be a natural kind, regardless of whether we know its essential property or not. In the case of the latter, talks of essential property are not that simple. For, a causal relation is dispositional and is exemplified under certain appropriate conditions. Dispositions of an ice-cube cooling the water in a glass and of the water melting the ice-cube can be instantiated between causally relational events. These causal events are mutual in the way that they call for each other and we can access to references in their causal interaction by identifying essential properties embedded in their causal interactions.

\subsection{Yinyang Causation, Non-dualistic and Non-symmetric}

Some people may wonder why those sympathetic with Humean causation cannot avoid dualism or physicalism and in what way yinyang causation allows philosophers to avoid dualism or physicalism. This question can be met by invoking two different notions of matter which two metaphysics each hold. As Humean causation is understood in terms of notions like contiguity, constant conjunction, and temporal priority, it presupposes that the causal relata are objects or physical events which are material, inert, and passive. This is the view of matter which is based on Cartesian dualism. But yinyan causation is explained in terms of notions like mutuality and simultaneity, having allowed the causal relata to be an agent of its own. The yinyang metaphysics is integrational, going against dualism. Of course, yinyang causation would be ready to allow a linear causation to be applied for certain special purposes. But this kind of flexibility is not expected from dualistic metaphysics.

Integrational causation is said to be mutual and simultaneous. Then, could not it be symmetric as well? Of course, certain relations which are mutual and simultaneous are symmetric. The relation of brother would be an example for it. Since two brothers, though they are a full person, do not have any agency to play a role in their being brothers, brotherhood is symmetric. But the causal relata in causal relations have different agencies, each of which plays its own roles such as selecting a partner, imputing its proper influence, and taking a direction. The causal relata in integrational causation are mutual and simultaneous but it cannot be symmetric. 


\section{Notes}

1. I prefer to write "yinyang" rather than "Yin-Yang" for the following reason: the yinyang ontology is so integrational that it is hard to separate clearly what is called "Yin" principles from what is called "Yang" principles and even harder to classify particular powers of properties or objects into Yin or Yang.

2. James Legge translated “誠 (cheng)” as “sincerity” (Confucius 1971). The translation may not be completely wrong since the notion has a distinct moral implication. But it is not balanced to convey the metaphysical thrust the notion intended to convey. This is the basis by which I propose to translate it as "integration."

3. The contrast between two forms of naturalism in naturalized intentionality and naturalized epistemology may help to understand the notion of naturalized reference. Yinyang ontology of naturalized intentionality regards all beings as natural agents whereas Quine's ontology of naturalized epistemology takes all non-human beings as inert objects. They both share a common element of naturalism, that is, the natural closure that events are determined by natural laws (Won, C. 2013, 96-107). But they differ in pursuing how natural laws are to be approached. Yinyang naturalism understands natural laws as an interpretation of a part of what is naturally and organically interrelated whereas Quine's naturalism takes natural laws as a scientific construction of a hypothesis to explain some of what are there naturally and quantifiably given. Then, there are senses in which reference 1 may be a result of construction of a hypothesis and reference 2 may be a naturally given.

4. The notion of information localization seems to have helped Fodor (1983) and Robbins (2009) advance the concept of informational encapsulation, but this concept fails to explain the connection among information encapsulations at the next stage because of its "dissociative" character.

\section{Works Cited}

Bacon, John, Keith Campbell, and Lloyd Reinhardt, eds. Ontology, Causality, and Mind: Essays in Honour of D. M. Armstrong. Cambridge: Cambridge University Press, 2008.

Chang, Chit-Yee. "Combinatorial Explosion, Information Encapsulation, and the Massive Modularity Hypothesis." The 1st CCPEA. Academia Sinica in Taipei, September 7-9, 2012. 33-34.

Chung, Daihyun. “Integrationality (誠): A Metaphysical Basis for the Concept of Causation” (CCPEA 2012 Teipei). The Korean Society of Analytic Philosophy, ed. Philosophical Analysis 17 (1): 1-20.

---. “Intentionality of Cheng (誠): Toward an Organic View.” Korean Philosophical Association, 2008a. 33-40.

---. “Fitting: A Case of Cheng (誠) Intentionality.” XXII World Congress of Philosophy. 2008b. 109.

---. "Yinyang: Toward Its Organic Conception.” (in Korean). The Korean Society of Analytic Philosophy, ed. Philosophical Analysis 4 (March 2001): 1-22.

Church, George M. and Ed Regis. Regenesis: How Synthetic Biology Will Reinvent Nature and Ourselves. New York: Basic Books, 2012.

Confucius. Analects, the Great Learning and the Doctrine of the Mean. Trans. Legge, James. New York: Dover, 1971/1893.

Floridi, Luciano. “Semantic Conception of Information.” Stanford Encyclopedia of Philosophy. 2013 Edition. Edward N. Zalta, ed. $\mathrm{URL}=<\mathrm{http} / /$ plato.stanford.edu/entries/information-semantic/>.

Fodor, Jerry A. The Modularity of Mind. Cambridge: MIT Press, 1983.

Godfrey-Smith, Peter and Kim Sterelny. "Biological Information.” Stanford Encyclopedia of Philosophy. 2007 Edition. Edward N. Zalta, ed. URL $=<$ http://plato.stanford.edu/entries/information-biological/>.

Heil, John. From an Ontological Point of View. Clarendon: Oxford University Press, 2003.

---. “Powerful Qualities.” Marmodoro. London: Routledge, 2010. 58-71.

Hume, David. Enquiries Concerning the Human Understanding and Concerning the Principles of Morals. Oxford: Clarendon, 1966/1777.

---. A Treatise of Human Nature. Ed. Louis Amhearst Selby-Bigge. Oxford: Clarendon, 1951/1888.

Kim, Jungoh. Study of Minds (in Korean). Seoul: Sigmapress, 2011.

Korean Philosophical Association. Philosophy and Culture: Metaphysics. 2008.

Mackie, J. L. Causes and Conditions, Causation and Conditionals. Ed. Ernest Sosa. Oxford University Press, 1975.

Marmodoro, A, ed. The Metaphysics of Powers. London: Routledge, 2010.

Marmodoro, A. “Introduction.” Marmodoro. 2010. 1-7.

Martin, Charles Burton. "Power for Realists.” Bacon, Campbell, and Reinhardt. 2008. 175-85.

Menzies, Peter. “Laws of Nature, Modality and Humean Supervenience.” Bacon, Campbell, and Reinhardt. 2008. 195-214. 
Molnar, George. Powers-A Study in Metaphysics. Oxford: Oxford University Press, 2003.

Mumford, Stephen and Rani Lill Anjum. “A Powerful Theory of Causation.” Marmodoro, ed. 2010. 143-57.

---. Getting Causes from Powers. Oxford: Oxford University Press, 2011.

Pollack, Andrew. “Scientists Add Letters to DNA’s Alphabet, Raising Hope and Fear.” New York Times. MAY 7, 2014.

Robbins, Phillip. "Modularity of Mind.” The Stanford Encyclopedia of Philosophy. 2009 Edition. Edward N. Zalta, ed. $\mathrm{URL}=<$ http://plato.stanford.edu/entries/modularity-mind/>.

Schrenk, Markus. “Antidotes for Dispositional Essentialism.” Marmodoro, ed. 2010. 169-79.

Schrödinger, Erwin. What Is Life? Cambridge: Cambridge University Press, 1944.

Shani, Itay. "Is There a Non-psychological Intentionality?” Metaphysics of Mind Workshop. Kyunghee University, May 30-31, 2012.

Williams, Neil E. “Puzzling Powers: The Problem of Fit.” Marmodoro, ed. 2010. 84-105.

Won, Chiwook. "Reasons, Actions, and Causes.” Brown University, Dissertation. 2013.

---. “Over Determination, Counterfactuals, and Mental Causation.” Philosophical Review 123.2 (2014): 205-29. 